Notre Dame Journal of Formal Logic

Volume XXI, Number 1, January 1980

NDJFAM

\title{
A CLASS OF TWO-PLACE THREE-VALUED UNARY GENERATORS
}

\author{
J. C. MUZIO and D. M. MILLER
}

1 Introduction A unary generator is a functor which may be used to define any one-place functor by the substitution of its arguments from a prescribed substitution set, the generator being used once in each definition with no negation of its output. In this paper, we examine the class of twoplace three-valued functors which are unary generators relative to the substitution set consisting of the constants, the variable, and the Post negations of the variable. Necessary and sufficient conditions for a functor to belong to this class are established and it is shown the class consists of 216 functors which are equivalent under a set of five transformations.

A universal decision element is one which may be used to define any of the functors of one or two arguments by the substitution of variables or constants in its arguments, the universal element being used exactly once in each definition. Sobocinski [11] has shown that for the two-valued case there exists such a functor with four arguments. The two-valued case has been extensively studied (see [1], [4], [7], [8], [9]). Rose [10], Loader [2], [3], and the authors [5] have presented three-valued universal decision elements. In a previous paper, the authors [6] have given a seven-place threevalued functor which acts as a universal decision element. This functor is a composition of a generalized condition disjunction and three identical functors from the class considered in this paper. The same composition applies to each member of the class resulting in a family of functors each behaving as a three-valued universal decision element.

Loader [2] has presented an alternative definition of a universal decision element. The functors considered here are-in his terminology-firstorder universal decision elements of the third degree. Loader has shown that for a two-place three-valued functor to be a unary generator the substitution set must contain at least two nontrivial one-place functors. He has further shown, by ennumeration on a computer, that for a substitution set with two nontrivial one-place functors, the Post negations result in 216 functors acting as unary generators as opposed to either zero or twelve for 
all other choices. Loader divided these 216 functors into eighteen equivalence classes by identifying two of the transformations presented below. There was no attempt to characterize the functors any further.

In the following discussion all functors and functions are three-valued. We use the term unary generator to mean a two-place three-valued unary generator relative to the substitution set consisting of the constants, the variable, and the Post negations of the variable. 0,1,2 denotes both the three values and the three logical constants of the system. Let $E=\{0,1,2\}$ be the value set. A unary function $K x$ such that $K 0=p, K 1=q$ and $K 2=r$ is written $K x=(p q r)$. The Post negations are defined as $P x=(120)$ and $Q x=$ (201). Note $P P x=Q x, Q Q x=P x, P Q x=Q P x=x$. A two-place function $D x y$ is defined by the nine entries in the table.

\begin{tabular}{c|ccc}
$D x y$ & 0 & 1 & 2 \\
\hline 0 & $a$ & $b$ & $c$ \\
1 & $d$ & $e$ & $f$ \\
2 & $g$ & $h$ & $i$
\end{tabular}

Let $A=\{a, b, c, d, e, f, g, h, i\} . \quad D$ is of type $\left[n_{0}, n_{1}, n_{2}\right]$ if $n_{0}, n_{1}$, and $n_{2}$ are respectively the number of occurrences of 0,1 , and 2 in $A$. The weight of a set of elements $B \subseteq A$ is defined to be the number of distinct elements in $B$. This is denoted $\omega(B)$ or, if $B=\{p, q, r\}, \omega\{p, q, r\}$.

2 The Triples Generated by $D$ The 24 nonconstant unary functions are divided into the following 8 classes:

$$
\begin{aligned}
& \mathbf{E}_{1}=\{(012),(120),(201)\} \\
& \mathbf{E}_{2}=\{(102),(021),(210)\} \\
& \mathbf{E}_{3}=\{(001),(010),(100)\} \\
& \mathbf{E}_{4}=\{(002),(020),(200)\} \\
& \mathbf{E}_{5}=\{(110),(101),(011)\} \\
& \mathbf{E}_{6}=\{(112),(121),(211)\} \\
& \mathbf{E}_{7}=\{(220),(202),(022)\} \\
& \mathbf{E}_{8}=\{(221),(212),(122)\}
\end{aligned}
$$

Clearly if the table for $D$ contains at least one entry of each of 0,1 , and 2 then (000), (111), and (222) may be defined directly. It suffices to investigate the conditions under which $D$ can generate at least one function from each $\mathbf{E}_{i}, 1 \leqslant i \leqslant 8$, since if

$D a_{1} a_{2}=(p q r), p, q, r \in\{0,1,2\}$ and $a_{1}, a_{2} \in\{0,1,2, x, P x, Q x\}$,

then

$D a_{1}^{\prime} a_{2}^{\prime}=(q r p)$, where for $i=1,2, a_{i}^{\prime}=\left\{\begin{aligned} a_{i} & \text { if } a_{i} \in\{0,1,2\} \\ P a_{i} & \text { if } a_{i} \in\{x, P x, Q x\},\end{aligned}\right.$

and, similarly,

$D a_{1}^{\prime \prime} a_{2}^{\prime \prime}=(r p q)$, where $a_{i}^{\prime \prime}=\left\{\begin{aligned} a_{i} & \text { if } a_{i} \in\{0,1,2\} \\ Q a_{i} & \text { if } a_{i} \in\{x, P x, Q x\} .\end{aligned}\right.$ 
Since the functions in each $E_{i}$ need not be considered separately a common notation is used. The triple $\langle p q r\rangle$ denotes any of the unary functions $(p q r)$, $(q r p)$, and $(r p q)$. Two triples are distinct iff the functions they denote are in different $\mathbf{E}_{i}$ classes.

There are nine triples generated by $D$ as follows:

$$
\begin{aligned}
D 0 x & =\langle a b c\rangle \\
D 1 x & =\langle d e f\rangle \\
D 2 x & =\langle g h i\rangle \\
D x 0 & =\langle a d g\rangle \\
D x 1 & =\langle b e h\rangle \\
D x 2 & =\langle c f i\rangle \\
D x x & =\langle a e i\rangle \\
D x P x & =\langle b f g\rangle \\
D x Q x & =\langle c d h\rangle
\end{aligned}
$$

These nine triples are termed the generating set of $D$. The first three are called row triples, the next three are called column triples, and the last three are called diagonal triples. Clearly for $D$ to be a unary generator its generator set must include at least one triple from each $\mathbf{E}_{i}, 1 \leqslant i \leqslant 8$.

3 Transformations of Unary Generators There are five elementary transformations which when applied to a unary generator result in a unary generator. These transformations are denoted $T_{i}, 1 \leqslant i \leqslant 5$, and are defined as follows

$$
\begin{aligned}
& T_{1} D x y=D y x \\
& T_{2} D x y=S D x y \text { where } S \text { denotes some permutation operation over } \mathbf{E} \\
& T_{3} D x y=D P x y \text { or } D Q x y \\
& T_{4} D x y=D x P y \text { or } D x Q y \\
& T_{5} D x y=D S x S y \text { where } S \text { denotes some permutation operation over } \mathbf{E} .
\end{aligned}
$$

It is straightforward to verify that the application of any of these transformations to a unary generator will result in a unary generator since the generator set of the function is invariant under these transformations.

4 The Main Theorem Given the two-place three-valued function defined by the table

\begin{tabular}{l|lll}
$D x y$ & 0 & 1 & 2 \\
\hline 0 & $a$ & $b$ & $c$ \\
1 & $d$ & $e$ & $f$ \\
2 & $g$ & $h$ & $i$
\end{tabular}

define $U=\{a, e, i\}, \quad V=\{b, f, g\}, \quad W=\{c, d, h\}, \quad X=\{a, f, h\}, \quad Y=\{b, d, i\}$ and $Z=\{c, e, g\}$.

Theorem Dxy is a unary generator if and only if: (i) it is of type $[3,3,3]$; (ii) $\omega(X)=\omega(Y)=\omega(Z)=2$; and (iii) $\omega(U)+\omega(V)+\omega(W)=7$.

The proof is by five lemmas. The first three establish the necessity of these conditions while the fourth and fifth establish their sufficiency. 
Lemma 1 A unary generator is of type [3, 3, 3].

Proof: Suppose $p \in \mathrm{E}$ only occurs twice in $A$. Any two of $a, b, \ldots, i$ only occur together in at most one of the triples in the generator set. Consequently, the generator set cannot include the two distinct triples that include $p$ twice. There must thus be at least $3 p$ 's in $A$, hence a unary generator must be of type $[3,3,3]$.

QED

Lemma 2 A unary generator is such that $\omega(X)=\omega(Y)=\omega(Z)=2$.

Proof: It is easily shown that under the action of any of the elementary transformations the set of values $\omega(X), \omega(Y)$ and $\omega(Z)$ is invariant although their order may change. Since the application of $T_{3}$ and $T_{4}$ transformations can convert $X$ to either $Y$ or $Z$, it will suffice to prove that if $\omega(X) \neq 2$ the function cannot be a unary generator.

If $\omega(X)=1$ and $a=f=h=p, p \in \mathrm{E}$, then no triple containing a repetition of $p$ can be generated since there can be no other occurrence of $p$ in $A$ as the function must be of type $[3,3,3]$.

If $\omega(X)=3$ there is at least one $p \in \mathrm{E}$ such that $p$ occurs once in $X$, once in $Y$, and once in $Z$. Consequently the generator set contains either three triples containing two $p$ 's and at most three triples containing a single $p$, or one triple containing three $p$ 's and six triples containing a single $p$. Neither case satisfies the definition of a unary generator since the generator set must contain at least four triples containing a single $p$ and at least two triples containing a pair of $p$ 's.

QED

Lemma 3 A unary generator is such that $\omega(U)+\omega(V)+\omega(W)=7$.

Proof: From Lemmas 1 and 2, we know a unary generator is of type $[3,3,3]$ and $\omega(X)=\omega(Y)=\omega(Z)=2$. Within these constraints there are three possibilities:

1. $\omega(U)=\omega(V)=\omega(W)=3$

2. $\omega(U)=\omega(V)=\omega(W)=2$

3. one of $U, V$ or $W$ has weight 3 while the other two have weight 2, hence $\omega(U)+\omega(V)+\omega(W)=7$.

Suppose $\omega(U)=\omega(V)=\omega(W)=3$. Since a unary generator must generate six triples of weight two, and $\omega(U)=\omega(V)=\omega(W)=3$, each row and column must have weight two. There must be a row with two $p$ 's and a column with two $p$ 's where one of the $p$ 's is in common. By transformations $T_{3}$ and $T_{4}$ they may be transformed into the first row and the first column. Now $a=p$ and by Lemma $2, f=h$. Also by Lemma $2, b=d=p$ or $c=g=p$ since $\omega(Y)=\omega(Z)=2$ and $p$ already occurs once in $X$. If $b=d=p, c=g$ since $\omega(U)=\omega(V)=\omega(W)=3$. Similarly if $c=g=p, b=d$. In either case the function is symmetric and therefore is not a unary generator.

Suppose $\omega(U)=\omega(V)=\omega(W)=2$. Either $a=e, a=i$, or $e=i$. By trans formation $T_{5}$ we can arrange to have $a=e$. Suppose $a=e=p, p \in \mathrm{E}$. One of $\{c, f, g, h\}$ must take the value $p$. Suppose $c=p$. By Lemma $2, d=h=f$ and thus $b=g=i .\langle a d g\rangle=\langle b e h\rangle=\langle c f i\rangle$ and the function is not a unary 
generator since a unary generator can generate only three triples of weight three, and the triples of weight three generated cannot all be the same. Suppose $f=p$. By Lemma 2, $b=g=c$ and thus $d=h=i .\langle a d g\rangle=\langle b e h\rangle=$ $\langle c f i\rangle$ and as above the function is not a unary generator. By transformation $T_{1}, g=p$ or $h=p$ lead to similar contradictions.

QED

Lemma 4 Of the nine triples in the generating set of a functor of type $[3,3,3]$ such that $\omega(X)=\omega(Y)=\omega(Z)=2$, six have weight two and three have weight three.

Proof: Consider $p \in \mathrm{E}$. One of $X, Y$, or $Z$ must contain exactly two $p$ 's since $\omega(X)=\omega(Y)=\omega(Z)=2$ and each of $0,1,2$ occurs exactly three times in $A$. Every pair of elements from $A$ is in $X, Y, Z$ or one of the triples in the generator set of the functor. Since exactly one pair of $p$ 's occurs in $X$, $Y$ or $Z$ and there are three $p$ 's as the functor is of type $[3,3,3]$, the generator set contains two triples containing two $p$ 's. This is true for $p=0,1$, or 2 and there are thus six triples of weight two in the generator set of the functor. No triple in the generating set can contain three $p$ 's and hence be of weight one, since two $p$ 's must occur in $X, Y$, or $Z$. The three remaining triples in the generator set must thus be of weight three.

QED

Lemma 5 A functor satisfying the conditions: (i) the functor is of type $[3,3,3]$; (ii) $\omega(X)=\omega(Y)=\omega(Z)=2$; and (iii) $\omega(U)+\omega(V)+\omega(W)=7$; is $a$ unary generator.

Proof: From Lemma 4, the functor must generate six triples of weight two and three triples of weight three. To show the functor is a unary generator we must show no pair of triples of weight two are equal and the three triples of weight three are not all equal.

Suppose two triples of weight two are equal. There are three possibilities: a row and a column triple; a row and a diagonal triple; a column and a diagonal triple. The other choices of two rows, two diagonals, or two columns violate the condition that the functor is of type $[3,3,3]$.

Suppose a row and a column triple are equal. By transformations $T_{3}$ and $T_{4}$ they may be transformed into the first row and column. As a result $b=d$ and $c=g$. Also by Lemma $2, f=h$. Thus $\omega(V)=\omega(W)=3$ which is a contradiction.

Suppose a row and a diagonal are equal. By transformations $T_{3}$ and $T_{4}$, we need only consider the case where $\langle a b c\rangle$ and $\langle a e i\rangle$ are equal triples. Either $a=b=i=p$ or $a=c=e=p$. The cases $a=b=e=p$ and $a=c=$ $i=p$ are invalid since none of $X, Y$, or $Z$ contains two $p$ 's and hence at least one does not have weight two. If $a=b=i=p, c=e$ and by Lemma 2, $f=h$. By Lemma 2, $c \neq e$ and $c \neq g$, hence $f=g=h$ and $c=d=e$. This is a contradiction since no diagonal has weight three. The case of a column and diagonal being equal is equivalent to this case as a result of transformation $T_{1}$.

Suppose the three triples of weight three are equal. Since one of these triples must be a diagonal triple it follows that the others must be a row triple and a column triple since if two rows (or two columns) have weight 
three the third must also and there can only be three triples of weight three. By transformations $T_{3}$ and $T_{4}$ we need only consider $\langle a b c\rangle=\langle a d g\rangle=$ $\langle a e i\rangle$. Now $b=d=e$ and $c=g=i$. Since the function is type $[3,3,3]$, $a=f=h$ which is a contradiction of $\omega(X)=2$.

QED

5 The Number of Two-Place Three-Valued Unary Generators There are $\frac{9 !}{3 ! 3 ! 3 !}=1680$ three-valued two-place functors of type $[3,3,3]$. For each of $\{a, f, h\},\{b, d, i\}$, and $\{c, e, g\}$ there are three choices for which pair of elements are equal. The equal pair in $\{a, f, h\}$ is either equal to the singleton value in $\{b, d, i\}$ or the singleton value in $\{c, e, g\}$. Finally there are six choices for which values occur twice in $\{a, f, h\},\{b, d, i\}$ and $\{c, e, g\}$. This give $3 \times 3 \times 3 \times 2 \times 6=324$ functors such that $\omega(X)=\omega(Y)=\omega(Z)=2$.

For these 324 functors there are three possibilities:

1. $\omega(U)=\omega(V)=\omega(W)=2$

2. $\omega(U)=\omega(V)=\omega(W)=3$

3. $\omega(U)=\omega(V)=\omega(W)=7$.

Given $\omega(U)=\omega(V)=\omega(W)=3, \omega(X)=2$, there are then 6 choices for $U$. For each of these choices, there are 3 choices for $f, 2$ choices for $h$, and the rest of the entries are then fixed. This yields $6 \times 3 \times 2=36$ functors. Given $\omega(U)=\omega(V)=\omega(W)=2, \omega(X)=2$, there are then 18 choices for $U$. One of the elements of $U$, say $a$, is distinct from the other two. If $f=h$ it is not possible to assign $c, b, d$, and $g$ so the only possibilities are $a=f$ or $a=h$. For each of these there are only two ways to assign $c, b, d$, and $g$. This yields $18 \times 2 \times 2=72$ functors. Clearly, there are $324-36-72=216$ two-place three-valued unary generators.

\section{REFERENCES}

[1] Foxley, E., "Determination of the set of all four-variable formulae corresponding to universal decision elements using a logical computer," Zeitschrift für mathematische Logik und Grundlagen der Mathematik, vol. 10 (1964), pp. 302-314.

[2] Loader, J., "Second order and higher order universal decision elements in m-valued logic," Proceedings of the 1975 International Symposium on Multiple-Valued Logic (1975), pp. 53-57.

[3] Loader, J., Private Communication.

[4] Muzio, J. C., "Partial universal decision elements," Notre Dame Journal of Formal Logic, vol. XV (1974), pp. 133-140.

[5] Muzio, J. C. and D. M. Miller, "Decomposition of ternary switching functions," Proceedings of 1973 International Symposium on Multiple-Valued Logic (1973), pp. 156-165.

[6] Muzio, J. C. and D. M. Miller, “A ternary universal decision element," Notre Dame Journal of Formal Logic, vol. XVII (1976), pp. 632-637.

[7] Pugmire, J. M. and A. Rose, "Formulae corresponding to universal decision elements," Zeitschrift für mathematische Logik und Grundlagen der Mathematik, vol. 4 (1958), pp. 1-9. 
[8] Rose, A., "Nouvelle méthode pour déterminer les formules qui correspondent a des éléments universels de décision," Comptes Rendus hebdomadaires des Séances de l'Academie des Sciences (Paris), vol. 244 (1957), pp. 2343-2345.

[9] Rose, A., "The use of universal decision elements as flip-flops," Zeitschrift für mathematische Logik und Grundlagen der Mathematik, vol. 4 (1958), pp. 169-174.

[10] Rose, A., "Sur les éléments universels trivalents de décision," Comptes Rendus hebdomadaires des Séances de l'Academie des Sciences (Paris), vol. 269 (1969), pp. A1-A3.

[11] Sobociński, B., “On a universal decision element," The Journal of Computing Systems, vol. 1 (1953), pp. 71-80.

University of Manitoba

Winnipeg, Manitoba, Canada

and

University of New Brunswick

Fredericton, New Brunswick, Canada 